\title{
A Microstrip Ring Resonator for Soil Moisture Measurements
}

Kamal Sarabandi and Eric S. Li

\author{
Radiation Laboratory \\ Department of Electrical Engineering and Computer Science \\ The University of Michigan \\ Ann Arbor, MI 48109-2122
}

\begin{abstract}
Accurate determination of spatial soil moisture distribution and monitoring its temporal variation have a significant impact on the outcomes of hydrologic, ecologic and climatic models. Development of a successful remote sensing instrument for soil moisture relies on the accurate knowledge of the soil dielectric constant $\left(\epsilon_{\text {soil }}\right)$ to its moisture content. Two existing methods for measurement of dielectric constant of soil at low and high frequencies are, respectively, the time domain reflectometry and the reflection coefficient measurement using an open-ended coaxial probe. The major shortcoming of these methods is the lack of accurate determination of the imaginary part of $\epsilon_{\text {soir }}$. In this paper a microstrip ring resonator is proposed for the accurate measurement of soil dielectric constant. In this technique the microstrip ring resonator is placed in contact with soil medium and the real and imaginary parts of $\epsilon_{\text {soil }}$ are determined from the changes in the resonant frequency and the quality factor of the resonator respectively. The solution of the electromagnetic problem is obtained using a hybrid approach based on the method of moments solution of the quasi-static formulation in conjunction with experimental data obtained from reference dielectric samples. Also a simple inversion algorithm for $\epsilon_{\text {soil }}=\epsilon_{r}^{\prime}+j \epsilon_{r}^{\prime \prime}$ based on regression analysis is obtained. It is shown that the wide dynamic range of the measured quantities provides excellent accuracy in the dielectric constant measurement. A prototype microstrip ring resonator at L-band is designed and measurements of soil with different moisture contents are presented and compared with other approaches.
\end{abstract}




\section{Introduction}

Successful modeling and understanding of global change are dependent upon an improved understanding of climatic and hydrologic processes. Soil moisture and its spatial and temporal variations play an important role in both climatic and hydrologic models. Radar remote sensing of soil moisture has been an ongoing area of research over the past two decades. Characterization of dependence of dielectric constant of soil to its moisture content is an important step in this endeavor. In this paper the theoretical and experimental aspects of a field-portable device for accurate measurement of both the real and imaginary parts of soil dielectric constant are discussed.

Two commonly used methods for field measurement of dielectric constant of soil are the time domain reflectometry (TDR) and the reflection coefficient measurement using an open-ended coaxial probe. In the TDR method the dielectric constant of the soil medium is determined from the delay time of the reflected wave from the open ends of a two-wire transmission line buried inside a soil medium [1]. The time delay can only provide the information about the real part of dielectric constant $\epsilon_{r}^{\prime}$. The accuracy of the TDR method is limited by the resolution of the TDR system. In the coaxial probe method [2], the complex dielectric constant is estimated from the measured complex reflection coefficient of the openended coaxial line pressed against the unknown material. The accuracy of this technique is limited by the small dynamic range of reflection coefficient as a function of $\epsilon_{r}^{\prime}$. Another problem with this technique when applied to soil medium is the small contact area of the coaxial tip which may be comparable to the soil particles. It should also be noted that soil dielectric measurement using coaxial probe is very sensitive to the applied pressure which degrades the accuracy of this measurement technique.

In this paper a microstrip ring resonator as a device for the dielectric measurement of soil is proposed to overcome the aforementioned problems with the existing devices. Application of ring resonators in characterizing the dielectric properties of materials is not a new idea $[3,4,5]$. However, these techniques are specialized for dielectric measurements of thin materials in a stripline configuration and/or only characterization of real part of low dielectric materials is considered. Since both the real and imaginary parts of the dielectric constant of soil have a wide dynamic range with respect to the soil moisture content and the measurement configuration is different from those reported in the literature, a careful theoretical and experimental study of the proposed problem is required. The dielectric measurement technique is very similar to the standard cavity measurement technique [6] with a subtle difference. Basically, the real and imaginary parts of the dielectric constant are to be inferred from the shift in the resonant frequency and the change in the quality factor of the resonator after placing the resonator in contact with the soil medium. The subtlety stems from the fact that the microstrip resonator can be regarded as a partially-filled resonant cavity and therefore the simple relationship between the dielectric constant and the measured changes in the resonant frequency and the quality factor can no longer be applied. Although the partially-filled nature of the microstrip resonators complicates the retrieval of dielectric constant from the measured quantities, it is a desirable feature in the soil dielectric constant measurements. In the standard cavity measurement, if the loss tangent of 
the dielectric material exceeds 0.05 , it becomes practically impossible to accurately measure the resonant frequency $\left(f_{0}\right)$ and the quality factor $(Q)$ of the loaded cavity. However, for the partially-filled resonators the changes in $f_{0}$ and $Q$ are substantially less affected by the loading dielectric material. It is shown that with appropriate microstrip resonator design, dielectric constant of soil with moisture content as high as $40 \%$ can easily be measured.

In Section 2 the theoretical analysis of the problem is given. In this analysis which will be referred to as the forward problem a numerical solution for calculating the resonant frequency and the quality factor of the resonator in terms of $\epsilon_{r}^{\prime}$ and $\epsilon_{r}^{\prime \prime}$ is provided. The numerical solution is constructed based on a quasi-static formulation. Since the practical microstrip resonators are constructed on finite substrate, the effect of finite substrate width is also investigated. In Section 3 the inverse problem is considered. There, a simple algorithm for retrieval of $\epsilon_{r}^{\prime}$ and $\epsilon_{r}^{\prime \prime}$ from the measured changes in $f_{0}$ and $Q$ based on regressional analysis is developed. Finally in Section 4 experimental results are presented. It is shown that the accuracy of the quasi-static solution degrades as the dielectric constant of the unknown material increases. This phenomenon has also been observed in stripline resonators [3]. In this section the theoretical results based on the quasi-static analysis are slightly modified to agree with experimental data obtained from reference dielectric samples. To verify the accuracy of this hybrid model, dielectric constant of a sandy soil with different moisture contents were measured using a prototype microstrip ring resonator and the results are compared with those measured by three other independent methods.

\section{Theory}

A microstrip ring resonator is a simple transmission line resonator whose geometry is shown in Fig. 1. The resonator is excited by a transmission line through a capacitive coupling and at certain frequencies, depending on the electrical length (perimeter) of the resonator, a standing wave pattern forms around the circular path of the resonator. The maximum voltage of the standing wave occurs at the exciting point. The resonant frequencies correspond to a condition where the perimeter of the ring is an integer multiple of the guided wavelength, that is,

$$
\lambda_{g}=\frac{\pi d}{n} \quad n=1,2,3, \cdots
$$

where $\mathrm{d}$ is the diameter of the ring and $\lambda_{g}$ is a function of the microstrip parameters $w_{s}, h$, $\epsilon_{s}$ and $\epsilon_{2}$ as shown in Fig. 2.

At resonant frequencies there exists a voltage maximum at $\frac{\pi d}{2}$ away from the excitation point. By placing a capacitively coupled transmission line at this voltage maximum point, the field in the resonator can be probed to detect the resonant frequencies. Basically the transmission coefficient $S_{21}$ of the two-port resonator is measured as a function of frequency and the resonant frequencies are identified as frequencies for which $\left|S_{21}\right|$ is maximized. It should be noted that the coupling capacitors tend to lower the resonant frequency and these must be as small as possible for accurate measurement of the resonant frequency. Spectral 
measurement of $\left|S_{21}\right|$ can also reveal the quality factor of the resonator which is a measure of power loss in the resonator. The dissipated power in the resonator includes the dielectric loss, the conductor loss and the radiation loss. For a given microstrip resonator with known substrate dielectric constant $\epsilon_{s}$, strip width $w_{s}$, and substrate height $h$ we are seeking an algorithm that would enable us to compute the complex dielectric constant of the half-space medium in contact with the ring resonator from the spectral measurement of $\left|S_{21}\right|$. To establish the relationship between the complex dielectric constant of the unknown medium and the measured guided wavelength and the $Q$ of the resonator, the quasi-static approximation for microstrip transmission lines is used. Strictly speaking microstrip structures cannot support TEM waves, however, at low frequency where the substrate height and strip width are small compared to the wavelength, it is expected that the quasi-static approximation provides accurate results [7]. The quasi-static approximations found in the literature are usually concerned with lossless dielectrics. In what follows a brief derivation of Poisson's equation for lossy dielectrics is given from which an integral equation for the charge distribution on the microstrip will be obtained. Starting from Maxwell's equations for time harmonic fields

$$
\begin{gathered}
\nabla \times H=j \omega \epsilon^{\prime} E+\sigma E, \\
\nabla \cdot D=\rho_{c}+\rho_{\text {imp }} \quad ; D=\epsilon^{\prime} E
\end{gathered}
$$

where $\rho_{c}$ is the conduction charge density, $\rho_{i m p}$ is the impressed charge density and $\epsilon^{\prime}$ and $\sigma$ are the permittivity and conductivity of the medium respectively. The conduction current $J=\sigma E$ can be related to the conduction charge density $\rho_{c}$ through the continuity relationship $\nabla \cdot J=-j \omega \rho_{c}$ and therefore

$$
\frac{j \sigma}{\omega} \nabla \cdot E=\rho_{c}
$$

Combining (3) and (2) and defining the complex permittivity as $\epsilon=\epsilon^{\prime}-\frac{j \sigma}{\omega}$, it can easily be shown that

$$
\epsilon \nabla \cdot E=\rho_{i m p}
$$

For TEM waves the electric and magnetic fields are irrotational and therefore the electric field can be obtained from a complex potential function $\phi$ through

$$
E=-\nabla \phi
$$

which together with (4) results

$$
\nabla^{2} \phi=-\frac{\rho_{i m p}}{\epsilon} .
$$

Assuming the ground plane and the strip of the microstrip are at potentials zero and $V_{0}$, (5) can be solved subject to the boundary conditions. In this case $\rho_{i m p}=\rho(x)$ is the induced charge distribution on the strip. If $G\left(x, y, x^{\prime}, y^{\prime}\right)$ represents the Green's function of the problem, then the complex potential function can be obtained from

$$
\phi(x, y)=\int_{\frac{-\omega}{2}}^{\frac{w}{2}} \rho\left(x^{\prime}\right) G\left(x, y, x^{\prime}, y^{\prime}\right) d x^{\prime} .
$$


Using the Fourier transform technique the Green's function for the microstrip problem is found to be

$$
G\left(x-x^{\prime}, y, h\right)=\frac{1}{\pi} \int_{0}^{\infty} \frac{e^{-\alpha(y-h)} \cos \left(\alpha\left(x-x^{\prime}\right)\right)}{\left(\epsilon_{s} \operatorname{coth}(\alpha h)+\epsilon_{2}\right) \alpha} d \alpha,
$$

where $\epsilon_{s}$ is the dielectric constant of the substrate and $\epsilon_{2}$ is the dielectric constant of the upper half-space. Since the potential function is known over the metalic strip, the integral equation for the induced charge density can be derived by evaluating the potential over the strip and is given by

$$
V_{0}=\int_{\frac{-\omega}{2}}^{\frac{\omega}{2}} \rho\left(x^{\prime}\right) G\left(x-x^{\prime}, h, h\right) d x^{\prime} .
$$

The integral equation can be solved numerically using the method of moments and point matching technique. Subdividing the strip into $N$ sufficiently small cells, (7) can be cast into a matrix equation

$$
\overline{\bar{Z}} \bar{R}=\bar{V}
$$

where $\bar{R}$ is the vector of unknown charge density and $\bar{V}$ is the excitation vector whose entries are constant $V_{0}$ potentials. To evaluate entries of $\overline{\bar{Z}}$, the Green's function given by (6) must be computed for different values of observation $\left(x_{m}\right)$ and source $\left(x_{n}\right)$ points. The decay rate of the integrand of (6) (when $y=h$ ) as a function of $\alpha$ is rather poor (the asymptotic behavior of the integrand for large values of $\alpha$ is $\frac{1}{\alpha\left(\epsilon_{0}+\epsilon_{2}\right)}$. To improve the convergence rate the order of integrations in (7) can be changed and the entries of the matrix are found to be

$$
Z_{m n}=\frac{2}{\pi} \int_{0}^{\infty} \frac{\sin \left(\alpha \frac{\Delta x}{2}\right) \cos \left(\alpha\left(x_{m}-x_{n}\right)\right)}{\alpha^{2}\left[\epsilon_{s} \operatorname{coth}(\alpha h)+\epsilon_{2}\right]} d \alpha
$$

where $\Delta x=\frac{w}{N}$. It is noted that the impedance matrix is Toeplitz and symmetric, that is, $Z_{m n}=Z_{p q}$ when $m-n=p-q$ and $Z_{m n}=Z_{n m}$. Thus integral in (8) must be evaluated only $N$ times to fully characterize the impedance matrix. Once the impedance matrix is characterized the charge density can be obtained from

$$
\bar{R}=\overline{\bar{Z}}^{-1} \bar{V} \text {. }
$$

The quantities of interest in the microstrip resonator are the electrical length and the quality factor of the resonator which are directly related to the line capacitance and conductance. The line capacitance is proportional to the total charge in phase with the voltage and the line conductance is proportional to the conduction current. The total complex charge on the strip represented by $Q=Q_{r}+j Q_{i}$ can be obtained from

$$
Q=\int_{\frac{-w}{2}}^{\frac{w}{2}} \rho(x) d x \simeq V_{0} \sum_{i} \sum_{j} Z_{i j}^{-1}
$$

from which the line admittance can be calculated and is given by

$$
Y=\frac{I}{V_{0}}=j \omega \frac{Q}{V_{0}}=j \omega \sum_{i} \sum_{j} Z_{i j}^{-1} .
$$


It can be shown that over a narrow range of frequency the microstrip line behaves like a capacitor in parallel with a resistor. The line capacitance and conductance thus are given by

$$
\begin{gathered}
C=\sum_{i} \sum_{j} \operatorname{Re}\left[Z_{i j}^{-1}\right] \quad F / m \\
G=-\omega \sum_{i} \sum_{j} \operatorname{Im}\left[Z_{i j}^{-1}\right] \quad S / m .
\end{gathered}
$$

For wideband applications a more complicated equivalent circuit must be considered. Assuming that the substrate material and the upper half-space medium are nonmagnetic, the line inductance becomes only a function of geometrical feature of the line and can be obtained from magnetostatic analysis. An alternative approach is to find the line capacitance of the air-filled microstrip line $\left(C_{0}\right)$ and find the line inductance from

$$
L=\frac{1}{C_{0} V_{p}^{2}}
$$

where $V_{p}=3 \times 10^{8} \mathrm{~m} / \mathrm{s}$ is the phase velocity of the air-filled line [7]. To check the validity and accuracy of the numerical method, the line capacitance computed by the method of moments with $\epsilon_{2}=1.0$ and $\epsilon_{s}=6.15$ is compared with the line capacitance computed using the conformal mapping technique and is shown in Fig. 3.

The quality factor of the resonator is defined as the ratio of the total average energy stored in the resonator to the power dissipated in a cycle. At resonance $W_{e}=W_{m}=\frac{1}{4} C V^{2}$, thus the quality factor due to the dielectric loss can be obtained from [6]

$$
Q_{d}=\omega \frac{W_{\mathrm{e}}+W_{m}}{P}=\omega \frac{\frac{1}{2} C V^{2}}{\frac{1}{2} G V^{2}}=\frac{\omega C}{G} .
$$

Another issue of practical importance is the finiteness of the substrate. The theoretical analysis given above is valid when the microstrip ground plane is of infinite extent. In practice the resonator substrate is finite and its effect on the line capacitance and conductance per unit length derived for microstrip with infinite ground plane must be investigated. Fig. 2 shows a microstrip with finite substrate and ground plane in contact with a half-space homogeneous medium. Our objective here is to characterize the smallest substrate width $w_{g}$ so that the difference between the line capacitance per unit length in this case and that of the infinite substrate is negligible. In this case a simple integral equation for the surface charge density on the strip and ground plane is obtained. The substrate dielectric is replaced with the induced polarization charge

$$
\rho_{p}=\epsilon_{0}\left(\epsilon_{s}-1\right)\left[\delta\left(\vec{r}-\overrightarrow{r_{c}}\right) \hat{n} \cdot E-\nabla \cdot E\right]
$$

where $\overrightarrow{r_{c}}$ is a position vector that specifies the contour of the substrate $(\mathcal{C})$ and $\hat{n}$ is an outward unit vector normal to the substrate boundary. After applying the Green's theorem 
the integral equation for the surface charge density and the complex potential function is found to be

$$
\begin{gathered}
\left(\frac{\epsilon_{o}+1}{2}\right) \phi(x, y)=-\epsilon_{0}\left(\epsilon_{s}-1\right) \int_{\mathcal{C}} \phi\left(x^{\prime}, y^{\prime}\right) \frac{\delta G\left(x, y ; x^{\prime}, y^{\prime}\right)}{\delta n^{\prime}} d c+\int_{-w_{s} / 2}^{w_{s} / 2} \rho_{s}\left(x^{\prime}\right) G\left(x, y ; x^{\prime}, 0\right) d x^{\prime} \\
+\int_{-w_{g} / 2}^{w_{s} / 2} \rho_{g}\left(x^{\prime}\right) G\left(x, y ; x^{\prime},-h\right) d x^{\prime}
\end{gathered}
$$

where $G\left(x, y ; x^{\prime}, y^{\prime}\right)$ is the Green's function of the half-space dielectric problems and is given by

$$
G\left(x, y ; x^{\prime}, y^{\prime}\right)=-\frac{1}{2 \pi \epsilon_{0}}\left[\ln \sqrt{\left(x-x^{\prime}\right)^{2}+\left(y-y^{\prime}\right)^{2}}-\frac{\epsilon_{2}-1}{\epsilon_{2}+1} \ln \sqrt{\left(x-x^{\prime}\right)^{2}+\left(y+y^{\prime}\right)^{2}}\right] .
$$

Once the integral equation is solved numerically the line capacitance and conductance per unit length can be obtained as shown before. Fig. 4 shows the line capacitance of a finite substrate microstrip line with $\epsilon_{s}=6.15, h=0.245 \mathrm{~cm}$, and $w_{s}=0.37 \mathrm{~cm}$ as a function of $w_{g} / w_{s}$ for $\epsilon_{2}=1$. It is shown that when $w_{g} / w_{s}>5$ the formulation based on infinite substrate is accurate for computation of the line capacitance.

\section{Inversion Algorithm}

In this section a retrieval algorithm for the complex dielectric constant of a half-space dielectric medium in contact with a microstrip ring resonator is sought. Following the standard cavity dielectric measurement approach the real and imaginary parts of the effective dielectric constant of the loaded resonator can be obtained from which the complex dielectric constant of the half-space medium must be inferred. Basically, first the resonant frequencies of the loaded (resonator in contact with the dielectric medium) and unloaded (resonator in

free space) resonators are found from the measured frequency spectrum of $\left|S_{21}\right|$. Then noting that at resonance the electrical length of the resonator is equal to the guided wavelength, it can easily be shown that:

$$
\frac{f^{u}}{f^{l}}=\frac{R e\left[\sqrt{\epsilon_{\text {eff }}^{l}}\right]}{\sqrt{\epsilon_{\text {eff }}^{u}}}
$$

where $f^{l}$ and $f^{u}$ are, respectively, the resonant frequencies of the loaded and unloaded resonators. Since the ring resonator is a transmission line resonator, the relationship between the effective dielectric constant and the line parameters can be obtained from:

$$
k_{0} \sqrt{\epsilon_{e f f}}=\omega \sqrt{L C\left(1-j \frac{G}{\omega C}\right)} .
$$

Assuming the substrate is lossless $\left(G^{u}=0\right)$ and noting that the line inductance is not a function of dielectric loading, it can be shown that

$$
C^{\prime}=C^{u} \frac{\epsilon_{e f f}^{\prime l}}{\epsilon_{e f f}^{u}}
$$




$$
\frac{G^{\prime}}{\omega^{\prime} C^{u}}=\frac{\epsilon_{e f f}^{\prime \prime \prime}}{\epsilon_{e f f}^{u}} .
$$

Comparing (17) to (11) and using (16), the imaginary part of the effective dielectric constant of the loaded resonator can be obtained from

$$
\epsilon_{e f f}^{\prime \prime \prime}=\frac{\epsilon_{e f f}^{\prime l}}{Q_{d}} .
$$

Using (15) and (18) the real part of the effective dielectric constant of the loaded resonator $\epsilon_{e f f}^{\prime \prime}$ in terms of measured $f_{f}^{\prime}$ and $Q_{d}$ and $\epsilon_{e f f}^{u}$ can be obtained. Once $\epsilon_{e f f}^{\prime \prime}$ and $\epsilon_{e f f}^{\prime \prime \prime}$ are obtained the line capacitance and conductance can be derived from (16) and (17).

Besides the dielectric loss, other factors such as radiation and conduction losses determine the quality factor of the resonator. If $Q_{u}$ represents the quality factor of the unloaded resonator and $Q_{d}$ is the quality factor due to the dielectric loss, the measured quality factor of the resonator $\left(Q_{m}\right)$ can be obtained from [6]

$$
\frac{1}{Q_{m}}=\frac{1}{Q_{u}}+\frac{1}{Q_{d}} .
$$

Equation (19) can be used to measure $Q_{d}$ when the radiation losses of the loaded resonator and unloaded resonator are the same or the radiation loss is negligible compared to the conductor and substrate losses. This can be verified by measuring the quality factor of the resonator when loaded with different lossless dielectrics. However, if the radiation loss is significant and varies with dielectric loading, (19) can be modified to

$$
\frac{1}{Q_{m}}=\frac{1}{Q_{u}}+\frac{1}{Q_{r}}+\frac{1}{Q_{d}}
$$

where $Q_{r}$ the quality factor due to radiation loss and its dependency to $\epsilon_{2}^{\prime}$ must be determined empirically. As will be shown later, for most soil conditions $Q_{d}<<Q_{u}$ and therefore characterization of $Q_{r}$ with respect to $\epsilon_{2}^{\prime}$ is not necessary.

The last step in the inversion algorithm is the computation of $\epsilon_{2}^{\prime}$ and $\sigma_{2}$ from the measured $C^{\prime}$ and $G^{\prime}$. In the previous section a numerical forward model for computation of the line capacitance and conductance in terms of $\epsilon_{2}^{\prime}$ and $\sigma_{2}$ was developed. Computation of the forward model for various $\epsilon_{2}^{\prime}$ and $\sigma_{2}$ reveals that $C^{l}$ and $G^{\prime}$ are smooth functions of $\epsilon_{2}^{\prime}$ and $\sigma_{2}$. Over the region of interest for $\epsilon_{2}^{\prime}$ and $\sigma_{2}$ these functions are one-to-one and onto which implies that the inverse functions exist. Since $C\left(\epsilon_{2}^{\prime}, \sigma_{2}\right)$ and $G\left(\epsilon_{2}^{\prime}, \sigma_{2}\right)$ are gentle functions of $\epsilon_{2}^{\prime}$ and $\sigma_{2}$, the inverse functions are also gentle functions of $C$ and $G$. To demonstrate this fact, $\epsilon_{2}^{\prime}$ and $\sigma_{2}$ versus the line capacitance and conductance of a microstrip with $\epsilon_{s}=6.15$, $h=0.245 \mathrm{~cm}$, and $w_{s}=0.37 \mathrm{~cm}$ are calculated numerically and are shown in Figs. 5 and 6. It is shown that the line capacitance is strongly dependent on $\epsilon_{2}^{\prime}$ and less influenced by $\sigma_{2}$ as expected. Also the line conductance is most sensitive to $\sigma_{2}$ and to a lesser extent to $\epsilon_{2}^{\prime}$. Therefore the inverse functions can simply be approximated by their Taylor series expansions, that is

$$
\begin{gathered}
\epsilon_{2} \approx a_{0}+a_{1} C+a_{2} G+a_{3} C G+a_{4} C^{2}+a_{5} G^{2}+a_{6} C G^{2}+a_{7} C^{2} G+a_{8} C^{3}+a_{9} G^{3} \\
+a_{10} C^{2} G^{2}+a_{11} C G^{3}+a_{12} C^{3} G+a_{13} C^{4}+a_{14} G^{4}
\end{gathered}
$$




$$
\begin{gathered}
\sigma_{2} \approx b_{0}+b_{1} C+b_{2} G+b_{3} C G+b_{4} C^{2}+b_{5} G^{2}+b_{6} C G^{2}+b_{7} C^{2} G+b_{8} C^{3}+b_{9} G^{3} \\
+b_{10} C^{2} G^{2}+b_{11} C G^{3}+b_{12} C^{3} G+b_{13} C^{4}+b_{14} G^{4}
\end{gathered}
$$

The coefficients of the polynomials can be obtained from the forward model using at least fifteen pairs of $(C, G)$. However, in order to find better estimate of the coefficients much more data points over the region of interest should be used in a least-mean-square estimator. Once the coefficients are determined the measured $C$ and $G$ can directly be used in (21) and (22) to find the unknowns $\epsilon_{2}^{\prime}$ and $\sigma_{2}$.

\section{Experimental Results}

In this section the design considerations for the development of a prototype microstrip ring resonator and some experimental results are given. Prompted by the need for a high quality factor transmission line resonator, the ring resonator as opposed to other types of transmission line resonators, such as a half-wavelength straight line resonator, was chosen. Another attractive feature of this resonator in measuring the dielectric constant of soil is its relatively large contact area with the soil medium. The coupling of energy to the resonator is accomplished using an unconventional method. Ring resonators are usually excited by a microstrip line through a capacitive air gap as shown in Fig. 7. However, for dielectric measurement applications it is better to excite the resonator with an open-ended coaxial line placed right underneath of the conducting strip as shown in Fig. 1. In this method the coupling capacitance of the resonator is not affected by the dielectric constant of the unknown material and therefore the quality factor due to the resonator coupling $\left(Q_{u}\right)$ remains unchanged. Another important design parameter is the resonator filling factor. This parameter signifies the percentage of the stored electric energy in the substrate to the overall stored energy. For example to measure very lossy dielectrics a large filling factor is required to keep most of the field lines inside the substrate. The filling factor is directly proportional to the line capacitance of the unloaded microstrip (inversely proportional to the unloaded characteristic impedance).

A microstrip ring resonator with a moderate filling factor that would allow accurate dielectric measurement of soil with volumetric moisture contents varying from $0 \%$ to $30 \%$ was designed using trial and error. It was found that a line with characteristic impedance of $50 \Omega$ would satisfy the filling factor requirements. For the prototype design, a Duroid 6006 substrate with $\epsilon_{s}=6.15$ and $h=0.254 \mathrm{~cm}$ was used. The strip width and the ring perimeter for the required unloaded characteristic impedance of $50 \Omega$ and resonant frequency of 1.25 $\mathrm{GHz}$ were calculated to be $w_{s}=0.37 \mathrm{~cm}$ and $\ell=11.35 \mathrm{~cm}$ respectively. The dimension of the finite substrate was chosen so that the constraint $w_{g} / w_{s}>5$ is satisfied for every point on the circular ring of the resonator. To examine the accuracy of the measurement technique and the aforementioned inversion algorithm, lossless reference dielectric samples were measured. An HP 8720 Network Analyzer with synthesized source was used to measure the quality factors and resonant frequencies reported in this paper. Six thick reference dielectric slabs (Stycast) with nominal dielectric constants of $2.2,3,6,10,13$, and 16 were measured using

the prototype resonator. It was found that the measurement accuracy degrades as the 
dielectric constant of the medium increases.

The experiments indicate that the quasi-static approximation overestimates the line capacitance when the dielectric constant of the half-space medium is increased. To investigate this phenomenon, two methods were pursued: (1) experimental, and (2) numerical. In the experimental investigation the effect of surface waves and the quasi-TEM nature of the approximate solution were examined. It is known that when the substrate height $h$ is large the structure becomes capable of supporting surface waves in the substrate. Three thinner substrates were used to construct ring resonators (all with characteristic impedance of $50 \Omega$ ) and the measurements with reference samples were repeated. Same amount of discrepancies were obtained independent of substrate thickness. Next we examined the effect of quasiTEM approximation in our formulation. For this purpose, a resonator was made from Duroid 6010 substrate with $\epsilon_{s}=10$ and used to measure the permittivity of the Stycast sample with dielectric constant 10 . In this case the medium surrounding the microstrip is homogeneous and the fundamental mode of propagation is TEM. However, a discrepancy in the measured dielectric on same order as before was observed. Having obtained inconclusive results from our experimental investigation, we resorted to numerical techniques. First a fullwave analysis for calculation of the effective propagation constant in microstrip lines based on the method of moments was used [8]. It was found that for the frequencies and microstrip dimensions used in this investigation the quasi-static solution provided very accurate results. Next the problem was analyzed completely using a three-dimensional FDTD numerical code [9]. After 20,000 iteration corresponding to 7 hours CPU time on a Cray super-computer, it was found that the resonant frequency obtained by the FDTD method had more discrepancy with the measured one than that obtained from the quasi-static method. One possible reason for the larger error is that the time domain methods may not be very suitable for resonant structures.

As a final resort we decided to rectify the problem by including an empirical correction factor in the quasi-static solution. Let us denote the line capacitances derived from the quasistatic solution and those measured from the inversion algorithm by $C_{q}$ and $C_{m}$ respectively. Fig. 8 shows the discrepancy in the line capacitance $\left(\Delta C=C_{q}-C_{m}\right)$ as a function of the six measured dielectric constants. Since the measured discrepancy is a smootn function of $\epsilon_{2}$ and no discrepancy exist when $\epsilon_{2}=1$ the following expression for the corrected line capacitance
is obtained:

$$
C_{c}=C_{q}-k\left(\epsilon_{2}^{\prime}-1\right),
$$

where $k=2.46 \times 10^{-12}$ is calculated using a least-mean-square linear estimator. This corrected line capacitance is then used in (21) and (22) to derive the coefficients $a_{1} \cdots a_{14}$ and $b_{1} \cdots b_{14}$. These coefficients are listed in Table 1 for a microstrip with $\epsilon_{s}=6.15, h=0.254 \mathrm{~cm}$,
and $w_{s}=0.37 \mathrm{~cm}$.

Next the behavior of the dielectric constant of a sandy soil as a function of moisture content is studied. The prototype ring resonator is used to measure soil samples with volumetric moisture contents ranging from $0 \%$ to $30 \%$. Fig. 9 shows typical responses of the ring resonator in contact with soil samples having moisture contents ranging from $0 \%$ to $30 \%$. Three other independent techniques were also used to measure the soil samples. These include the open-ended coaxial probe, cavity resonator, and air-filled coaxial line. The dielec- 
tric measurement using air-filled coaxial line is based on reflection coefficient measurement of a short-circuited coaxial line filled with soil samples. In this method the middle section of a long hollow coaxial line is used as the sample holder as shown in Fig. 10. Measuring the reflection coefficient over a wide bandwidth $(B)$ and using the time domain capability of the network analyzer, the reflected signal after traveling twice through the sample can be measured. The hollow section of the coaxial line must be longer than the spatial resolution $\left(3 \times 10^{8} / 2 B\right)$ of the system. Fig. 11 shows the steps involved in measuring the reflection coefficient of the short circuit for a $50 \Omega$ air-line of length $l=50 \mathrm{~cm}$ filled with $d=20 \mathrm{~cm}$ of dry sand. The measured complex reflection coefficient is related to the dielectric constant of the sample through

$$
S_{11}=-\left(1-R^{2}\right)^{2} e^{-j 2 k_{0} l} e^{-j 2 k_{0}\left(\sqrt{\epsilon_{\mathrm{r}}}-1\right) d},
$$

where $R=\frac{1-\sqrt{c_{r}}}{1+\sqrt{c_{r}}}$ is the reflection coefficient at the interface of air and soil. By inverting (24) the real and imaginary parts of the soil dielectric are obtained. The accuracy of this method decreases as the imaginary part of the dielectric constant increases unless the sample length is decreased.

Figs. 12 and 13 show the measured real and imaginary parts of the dielectric constants of the sandy soil with seven different moisture contents using the four independent measurement techniques. The cavity method could only be used for the $0 \%$ and $5 \%$ moisture conditions and shows an excellent agreement with the ring resonator measurement. For the real part of the dielectric constant the agreement among all four techniques is very good. However, it is shown that the coaxial probe overestimates the imaginary part of the dielectric constant as the moisture content increases.

\section{Conclusions}

Theoretical, numerical, and experimental aspects of a microstrip ring resonator based dielectric measurement device are discussed in this paper. The real and imaginary parts of dielectric constant of a homogeneous medium in contact with the ring resonator is calculated from the shift in the resonant frequency and the change in the quality factor of the resonator. The solution to the forward problem is obtained from a numerical analysis of the quasi-static solution. For the inverse problem simple algebraic expressions based on regression analysis of the forward problem are also obtained. Measurements of reference dielectric samples showed that the accuracy of the quasi-static solution decreases as the dielectric constant of the halfspace medium increases. To identify the source of error, full-wave numerical analysis and extensive experiments were conducted. Our efforts in identifying the source of errors were inconclusive. The errors in the quasi-static solution are corrected empirically from which the inversion expressions are obtained. The validity of the ring resonator measurement technique is demonstrated by comparing the measured dielectric constants of a sandy soil with different moisture contents with those obtained from three other measurement methods. It is shown that the measurement technique is both accurate and versatile. The ring resonator, because of its large contact area, is very suitable for dielectric measurement of soil and can easily be constructed into a field portable device. 
Acknowledgement: This project was supported by NASA under contact NAGW-2151.

\section{References}

[1] Topp, G.C. and J.L. Davis, "Measurement of Soil Water Content Using Time-domain Reflectrometry (TDR): A Field Evaluation", Soil Sci. Soc. Am. J., vol. 49, pp. 19-24,
1985.

[2] Ulaby, F.T., T.H. Bengal, M.C. Dobson, J.R. East, J.B. Garvin and D.L. Evans, "Microwave Dielectric Properties of Dry Rocks", IEEE Trans. on Geoscience and Remote Sensing, vol. 28, no.3, pp. 325-336, May. 1990.

[3] Bernard, P.A. and J. M. Gautray, "Measurement of Dielectric Constant Using a Microstrip Ring Resonator", IEEE Trans. on Microwave Theory and Techniques, vol. 39, no. 3, pp. 592-595, March 1991.

[4] Hoffman, R.K., Handbook of Microwave Integrated Circuits, Norwood, MA: Artech House, 1987.

[5] Vainikainen, P.V., E.G. Nyfors, and M.T. Fischer, "Radiowave Sensor for Measuring the Properties of Dielectric Sheets: Application to Veneer Moisture Content and Mass per Unit Area Measurement", IEEE Trans. Inst. Measurement, vol. 36, no. 4, Dec. 1987.

[6] Collin, R.E., Foundations for Microwave Engineering, New York: McGraw-Hill, pp 317329, 1966.

[7] Elliott, R.S., An Introduction to Guided Waves and Microwave Circuits, Englewood Cliffs, New Jersey: Prentice Hall, pp 61-67, 1993.

[8] Jackson, R.W. and D. M. Pozar, "Full Wave Analysis of Microstrip Open-end and Gap Discontinuities", IEEE Trans. on Microwave Theory and Techniques, vol. MTT-33, no. 10, pp. 1036-1042, Oct. 1985.

[9] Dib, N., Personal Communication. 

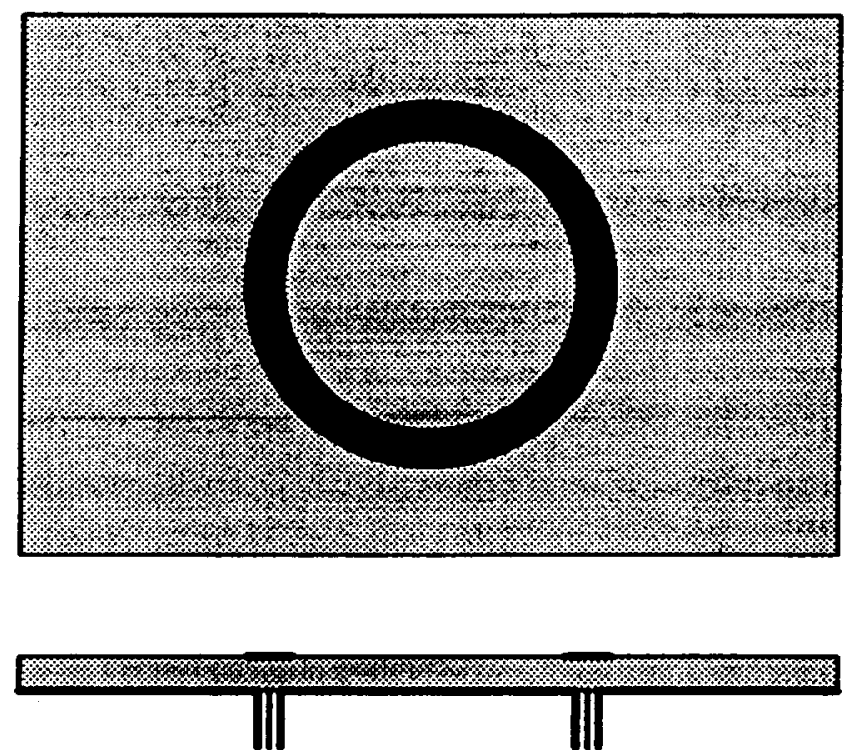

Figure 1: Top view and side view of a ring resonator used in this investigation.

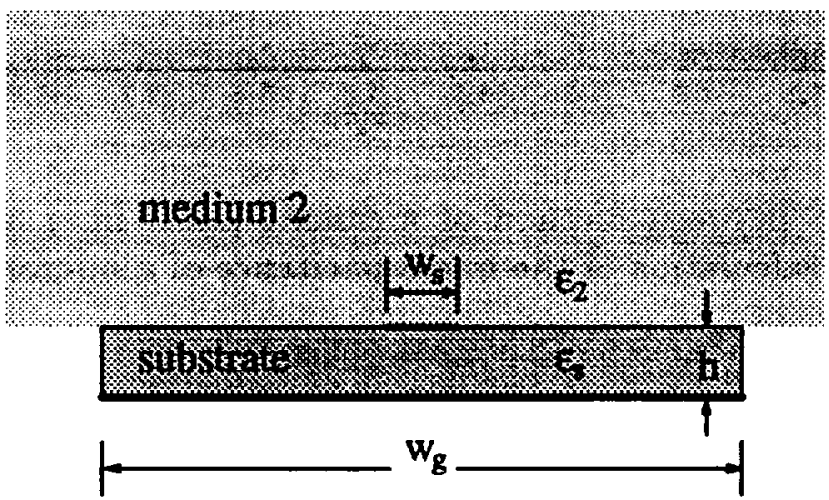

Figure 2: Geometry of a microstrip line in contact with a half-space homogeneous dielectric medium. 


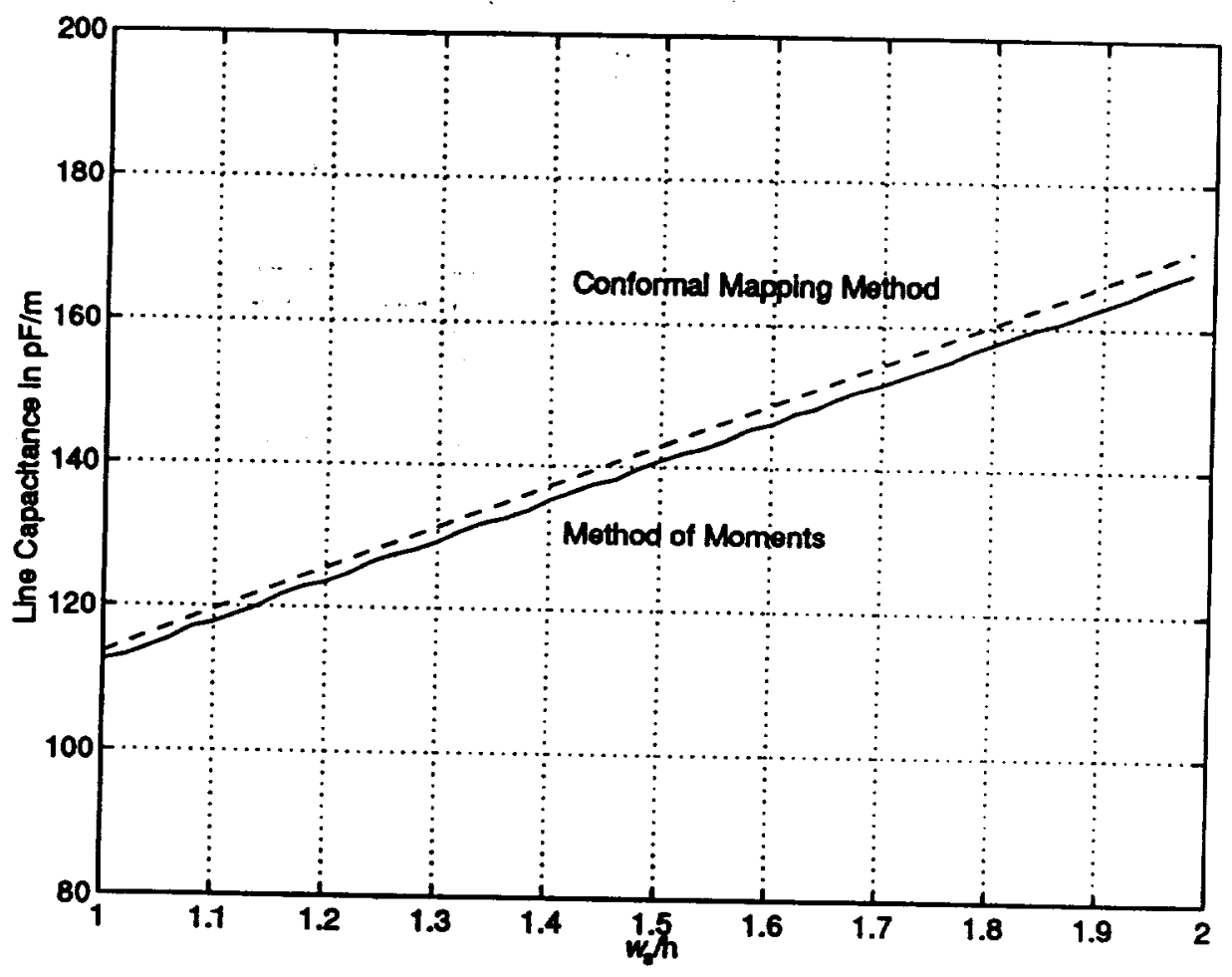

Figure 3: Comparison between method of mements (solid line) and conformal mapping method (dash line) in calculation of the line capacitance of a microstrip line with $\epsilon_{s}=6.15$. 


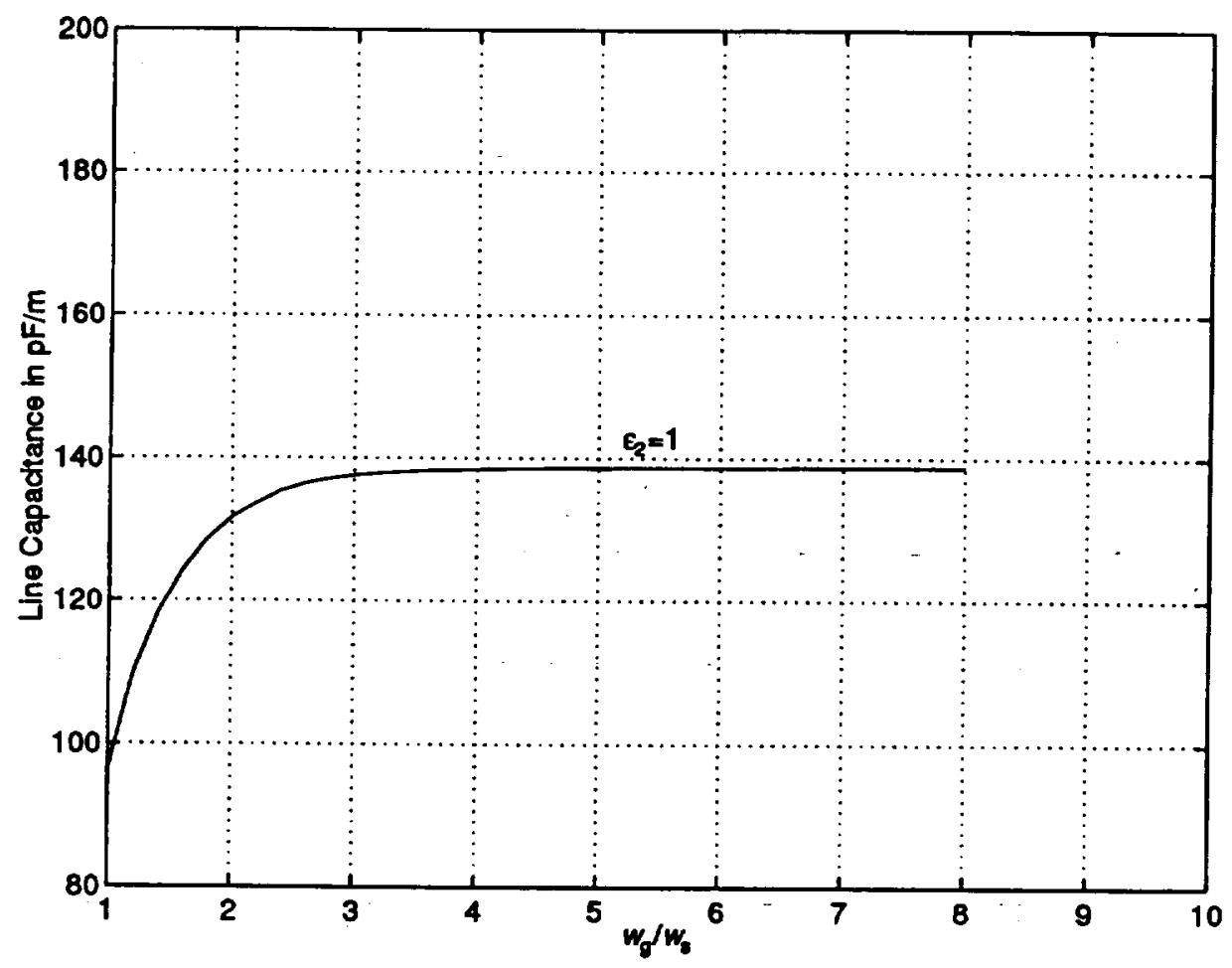

Figure 4: Line capacitance of a finite substrate microstrip line with $\epsilon_{s}=6.15$ and $w_{s} / h=1.46$ as a function of $w_{g} / w_{s}$. 


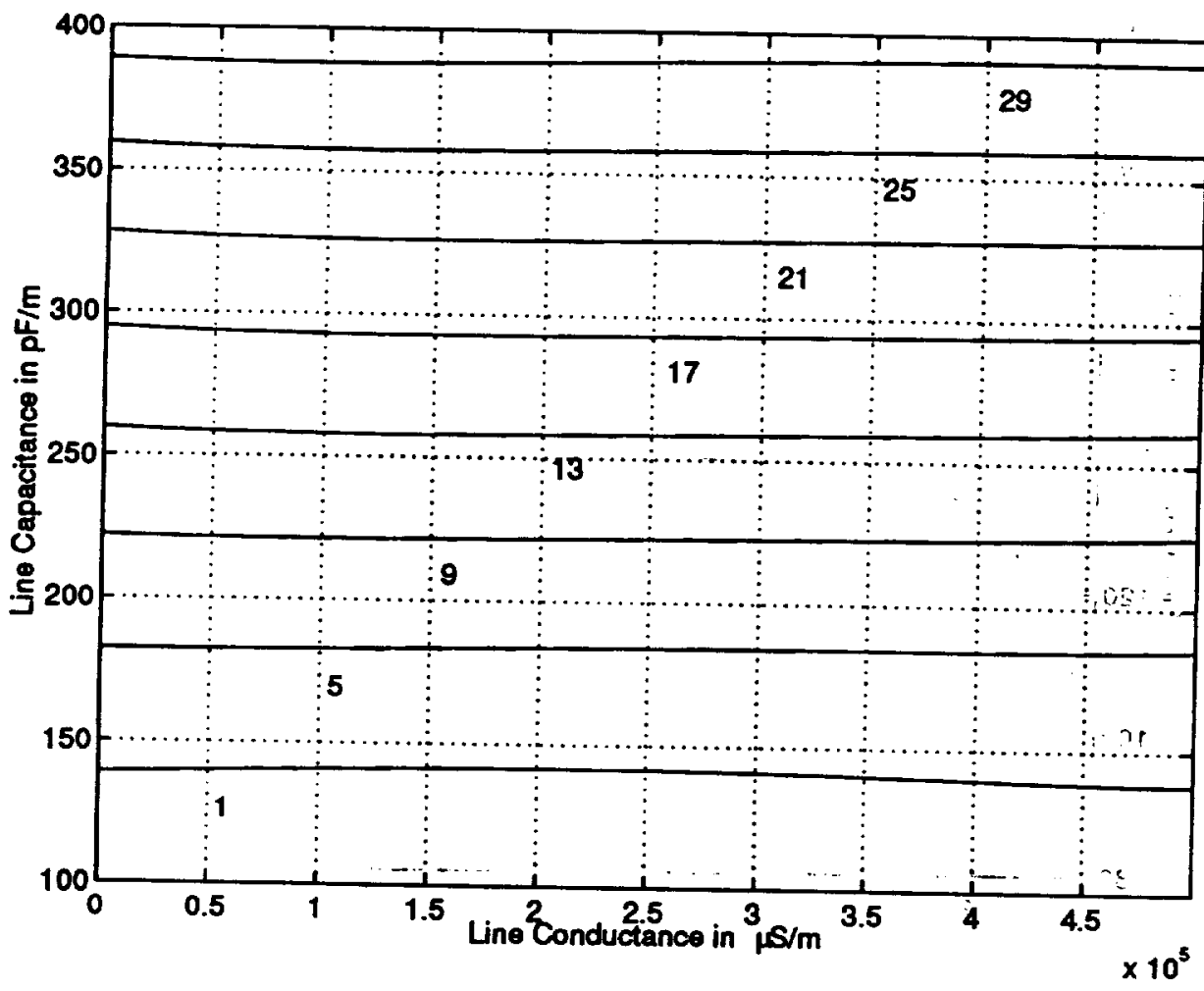

Figure 5: Contour map of the real part of dielectric constant as a function of line conductance and capacitance for a microstrip line with $\epsilon_{s}=6.15$ and $w_{s} / h=1.46$. 


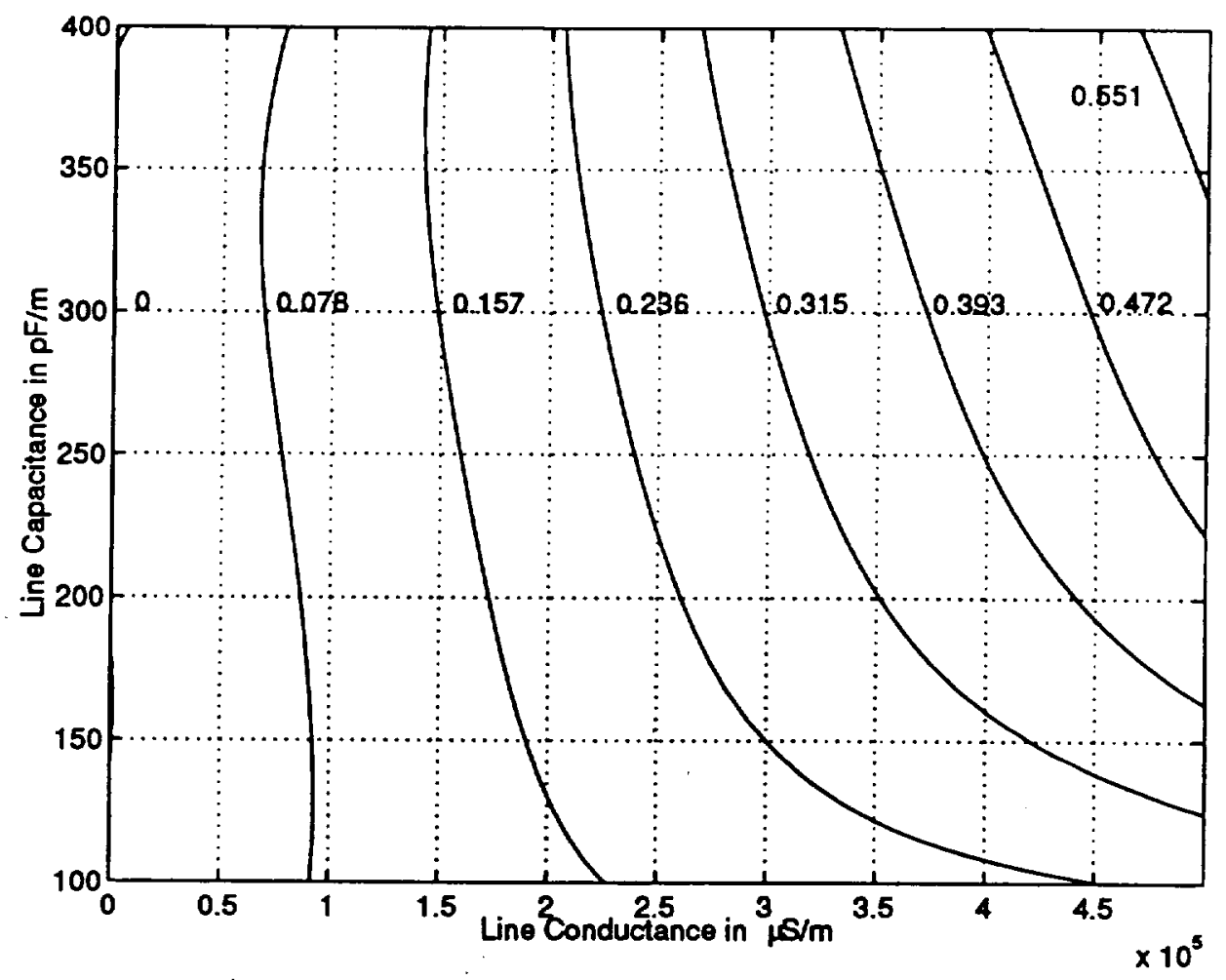

Figure 6: Contour map of conductivity as a function of line conductance and capacitance for a microstrip line with $\epsilon_{s}=6.15$ and $w_{s} / h=1.46$.
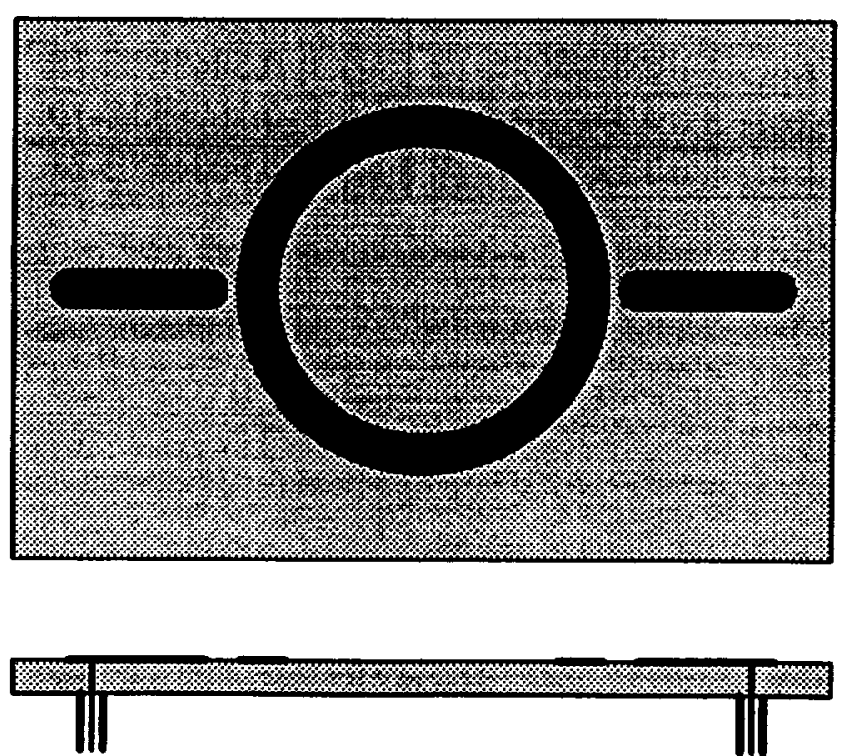

Figure 7: Top view and side view of a conventional ring resonator. 


\begin{tabular}{|r|r|r|r|}
\hline \multicolumn{2}{|c|}{$\epsilon_{2}^{\prime}$} & \multicolumn{2}{c|}{$\sigma_{2}$} \\
\hline \hline$a_{0}$ & $-0.787696 \mathrm{E}+01$ & $b_{0}$ & $0.333251 \mathrm{E}-01$ \\
\hline$a_{1}$ & $0.113820 \mathrm{E}-01$ & $b_{1}$ & $-0.419435 \mathrm{E}-03$ \\
\hline$a_{2}$ & $-0.625590 \mathrm{E}-06$ & $b_{2}$ & $0.619400 \mathrm{E}-07$ \\
\hline$a_{3}$ & $-0.170414 \mathrm{E}-07$ & $b_{3}$ & $0.907383 \mathrm{E}-08$ \\
\hline$a_{4}$ & $-0.437116 \mathrm{E}-03$ & $b_{4}$ & $0.755818 \mathrm{E}-06$ \\
\hline$a_{5}$ & $0.518208 \mathrm{E}-11$ & $b_{5}$ & $-0.924463 \mathrm{E}-12$ \\
\hline$a_{6}$ & $-0.858796 \mathrm{E}-13$ & $b_{6}$ & $0.863279 \mathrm{E}-14$ \\
\hline$a_{7}$ & $0.193313 \mathrm{E}-09$ & $b_{7}$ & $-0.346372 \mathrm{E}-10$ \\
\hline$a_{8}$ & $-0.465772 \mathrm{E}-06$ & $b_{8}$ & $0.610040 \mathrm{E}-08$ \\
\hline$a_{9}$ & $0.628353 \mathrm{E}-17$ & $b_{9}$ & $-0.300749 \mathrm{E}-18$ \\
\hline$a_{10}$ & $0.130853 \mathrm{E}-15$ & $b_{10}$ & $-0.121781 \mathrm{E}-16$ \\
\hline$a_{11}$ & $0.344406 \mathrm{E}-19$ & $b_{11}$ & $-0.313788 \mathrm{E}-20$ \\
\hline$a_{12}$ & $-0,386692 \mathrm{E}-12$ & $b_{12}$ & $0.504362 \mathrm{E}-13$ \\
\hline$a_{13}$ & $0.359810 \mathrm{E}-09$ & $b_{13}$ & $-0.167593 \mathrm{E}-10$ \\
\hline$a_{14}$ & $-0.123399 \mathrm{E}-22$ & $b_{14}$ & $2.0 .903452 \mathrm{E}-24$ \\
\hline
\end{tabular}

Table 1: Taylor polynomial coefficients for $\epsilon_{2}^{\prime}$ and $\sigma_{2}$ respectively. 


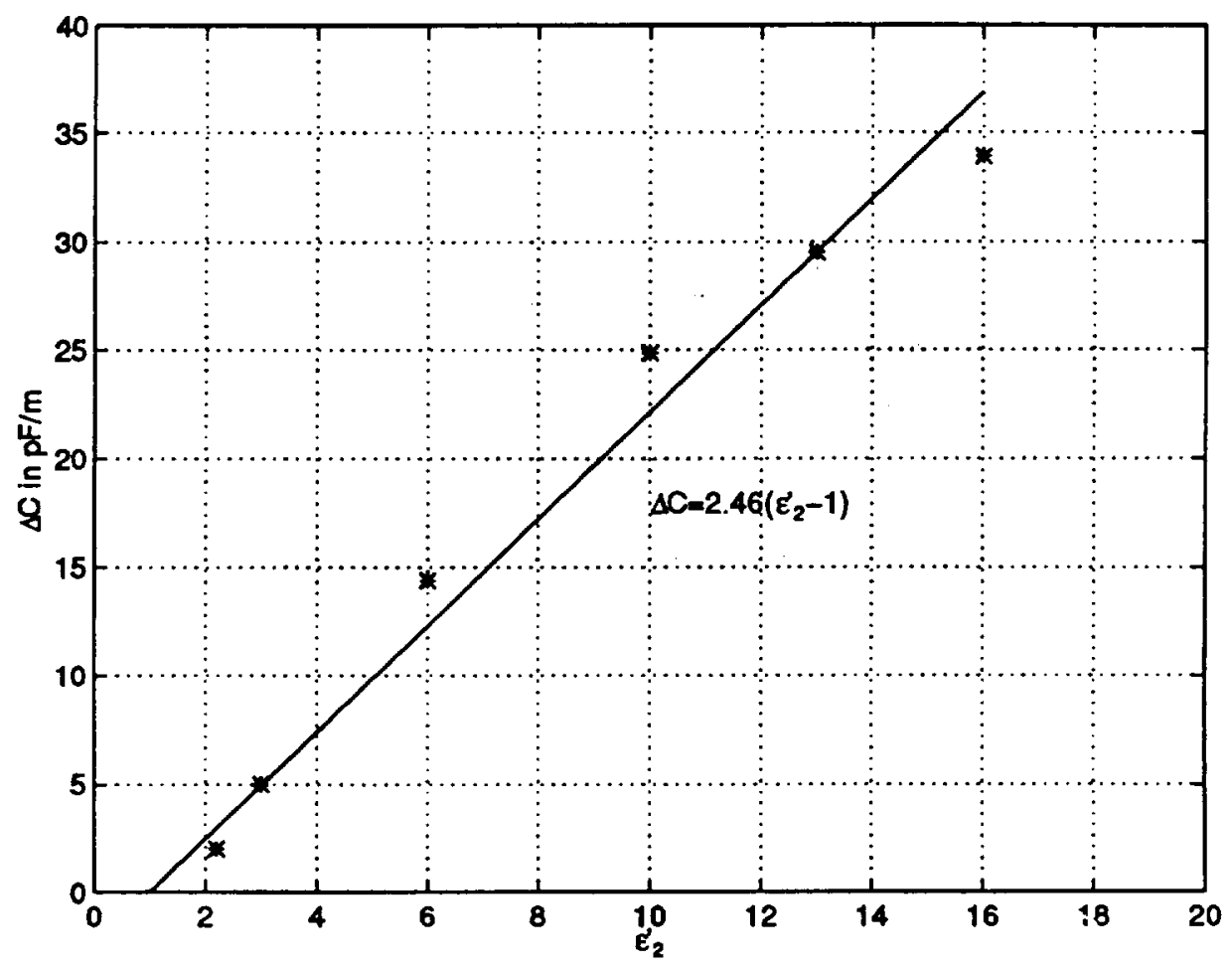

Figure 8: $\Delta C$ as a function of $\epsilon_{2}^{\prime}$. 


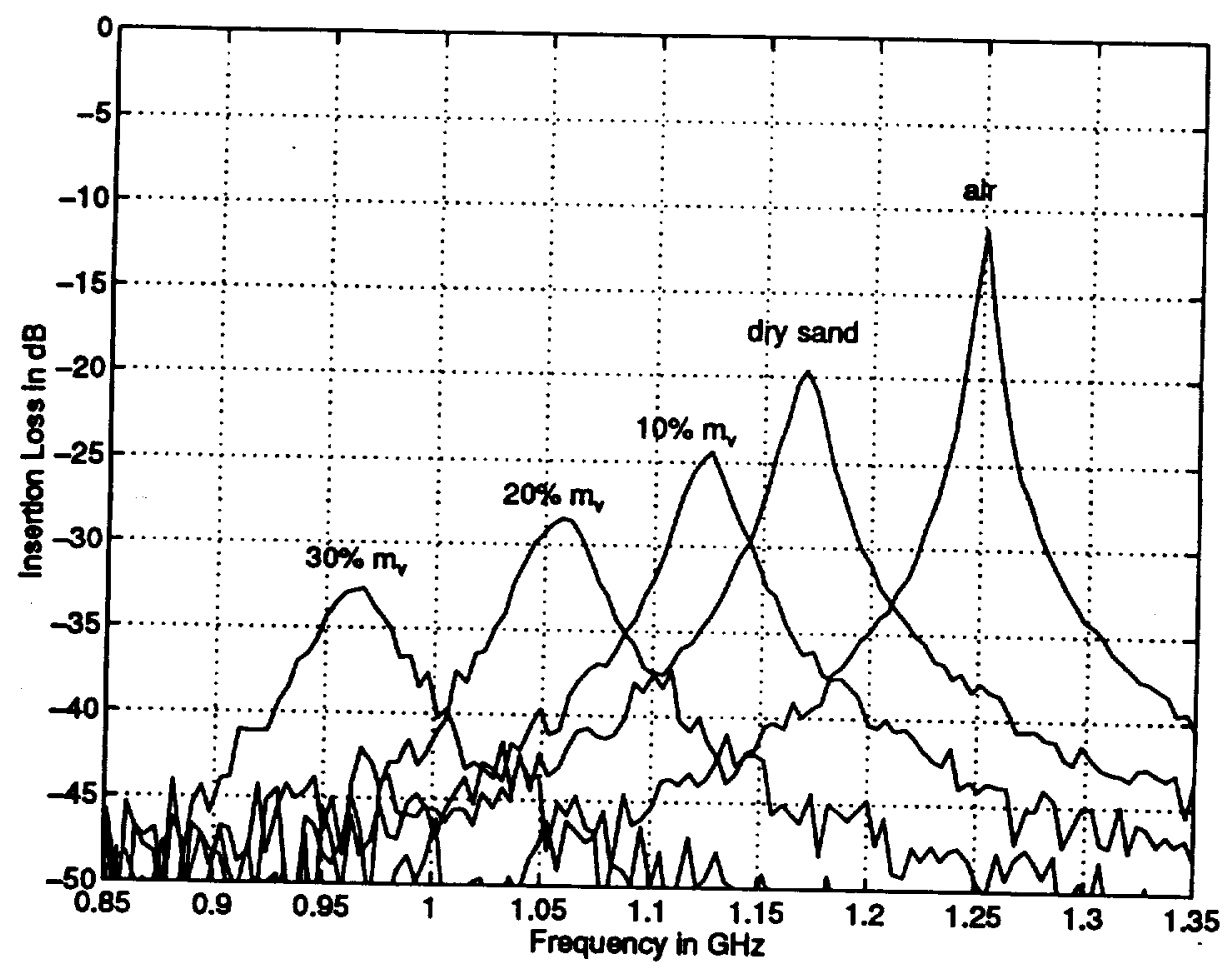

Figure 9: Sand moisture spectral response.

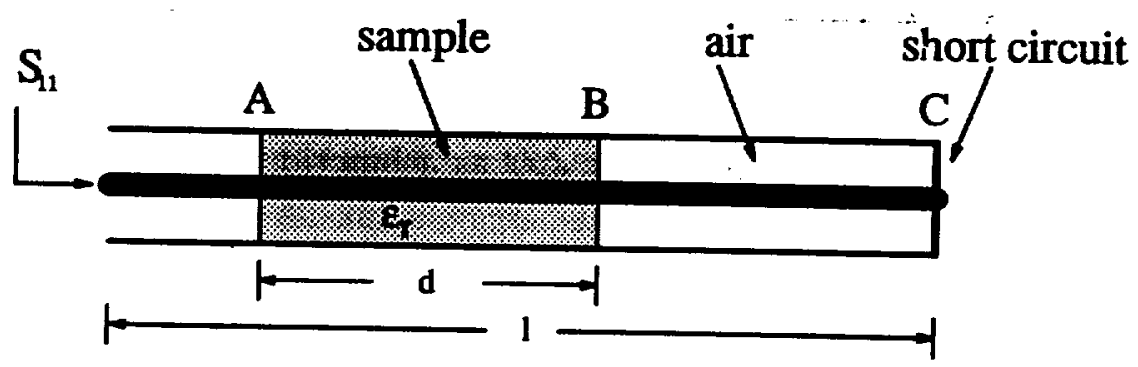

1st reflection

2nd reflection

3 rd reflection

Figure 10: The air-filled coaxial line sample holder. 
(a) Time Domain

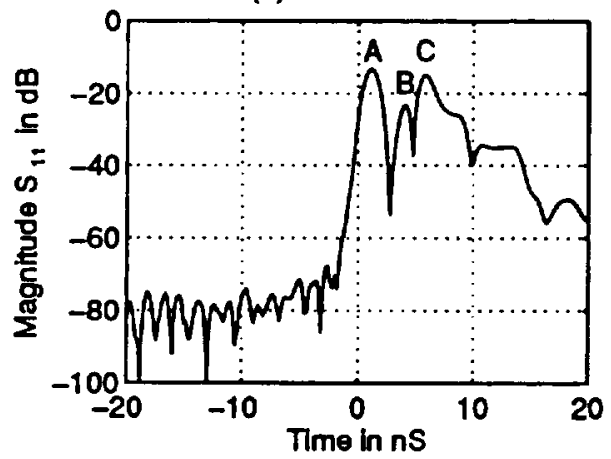

(c) Frequency Domain

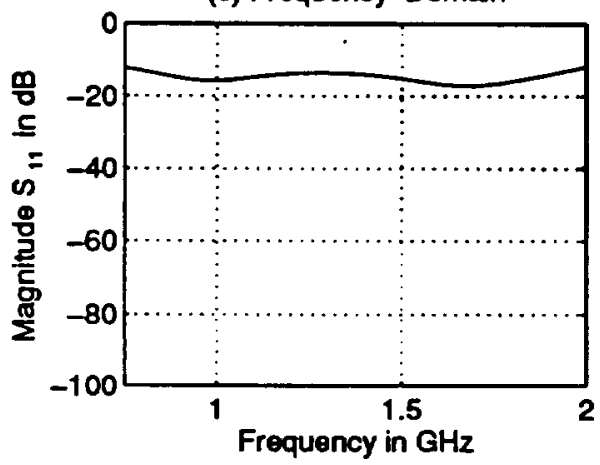

(b) Gated Response in Time Domain

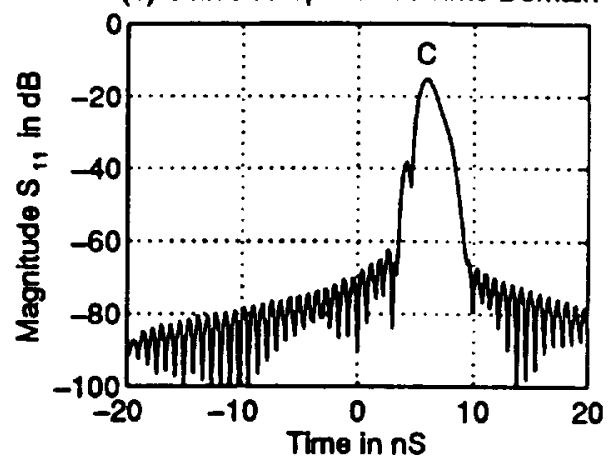

(d) Frequency Domain

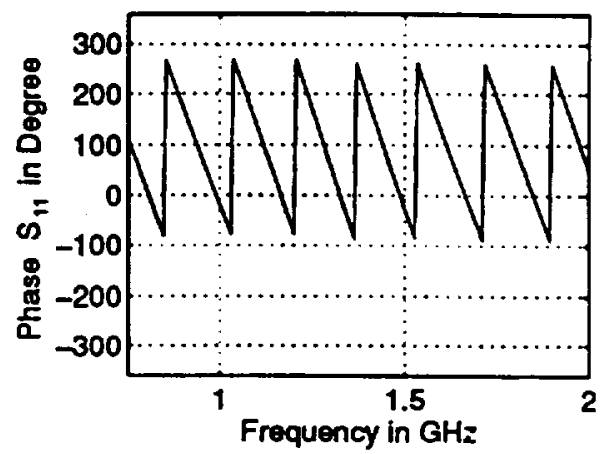

Figure 11: Steps in the measurement of the short circuit reflection coefficient: (a) time domain response of the total reflection coefficient, (b) time domain gated response of the short circuit, (c) magnitude and (d) phase of the short circuit reflecton coefficient. 


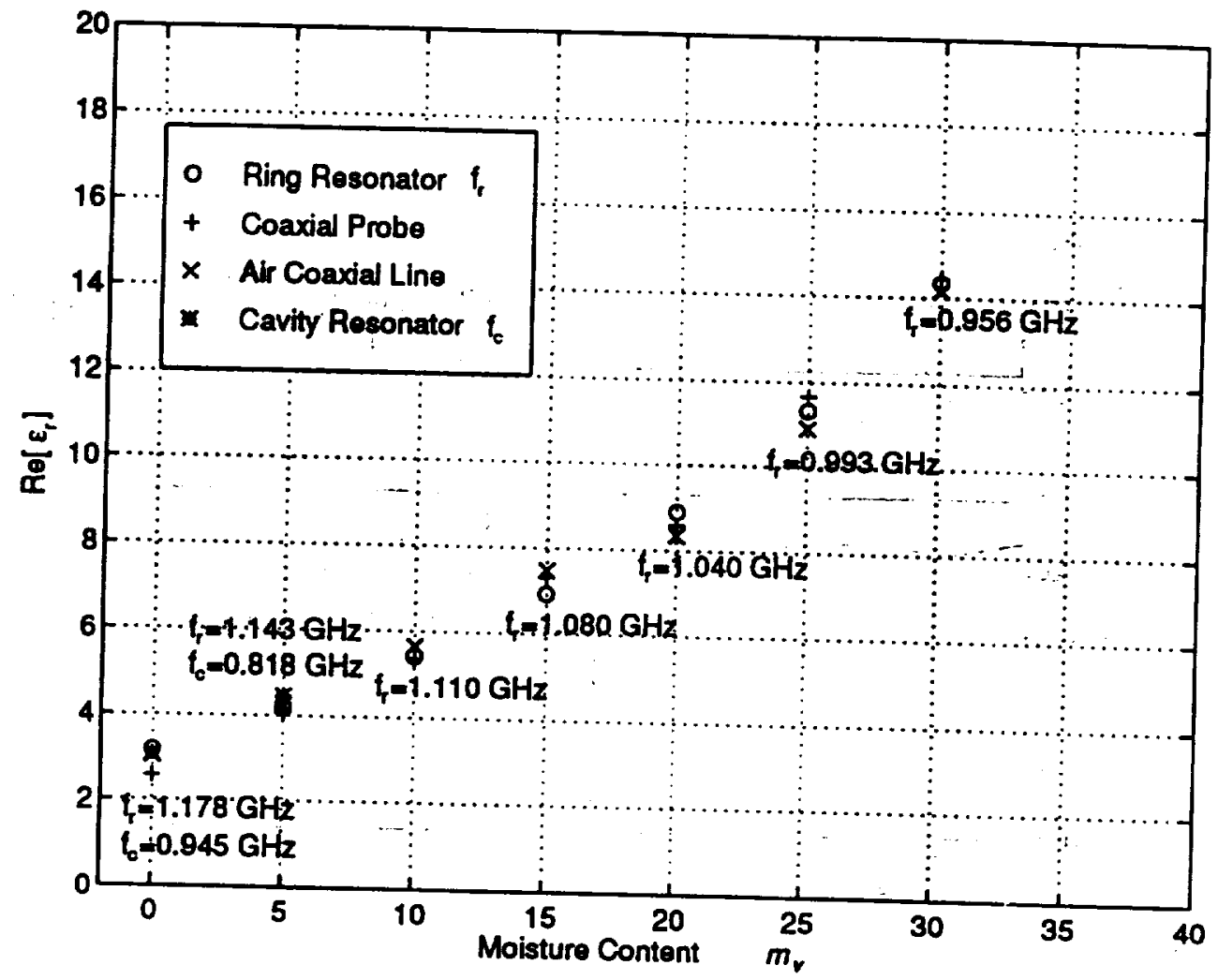

Figure 12: Different methods for measuring $\epsilon_{\tau}^{\prime}$ of a sandy soil with various moisture contents. 


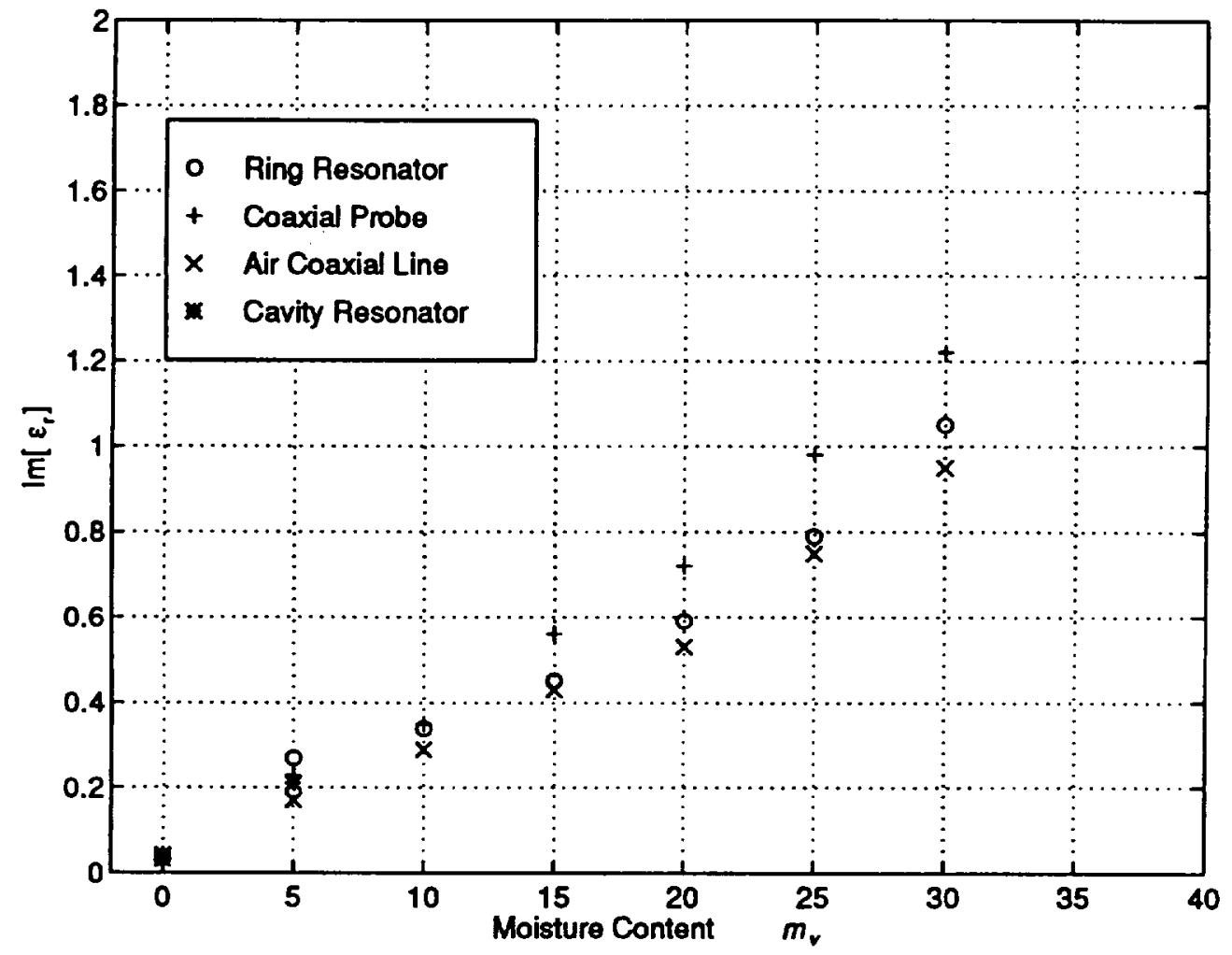

Figure 13: Different methods for measuring $\epsilon_{\tau}^{\prime \prime}$ of a sandy soil with various moisture contents. 


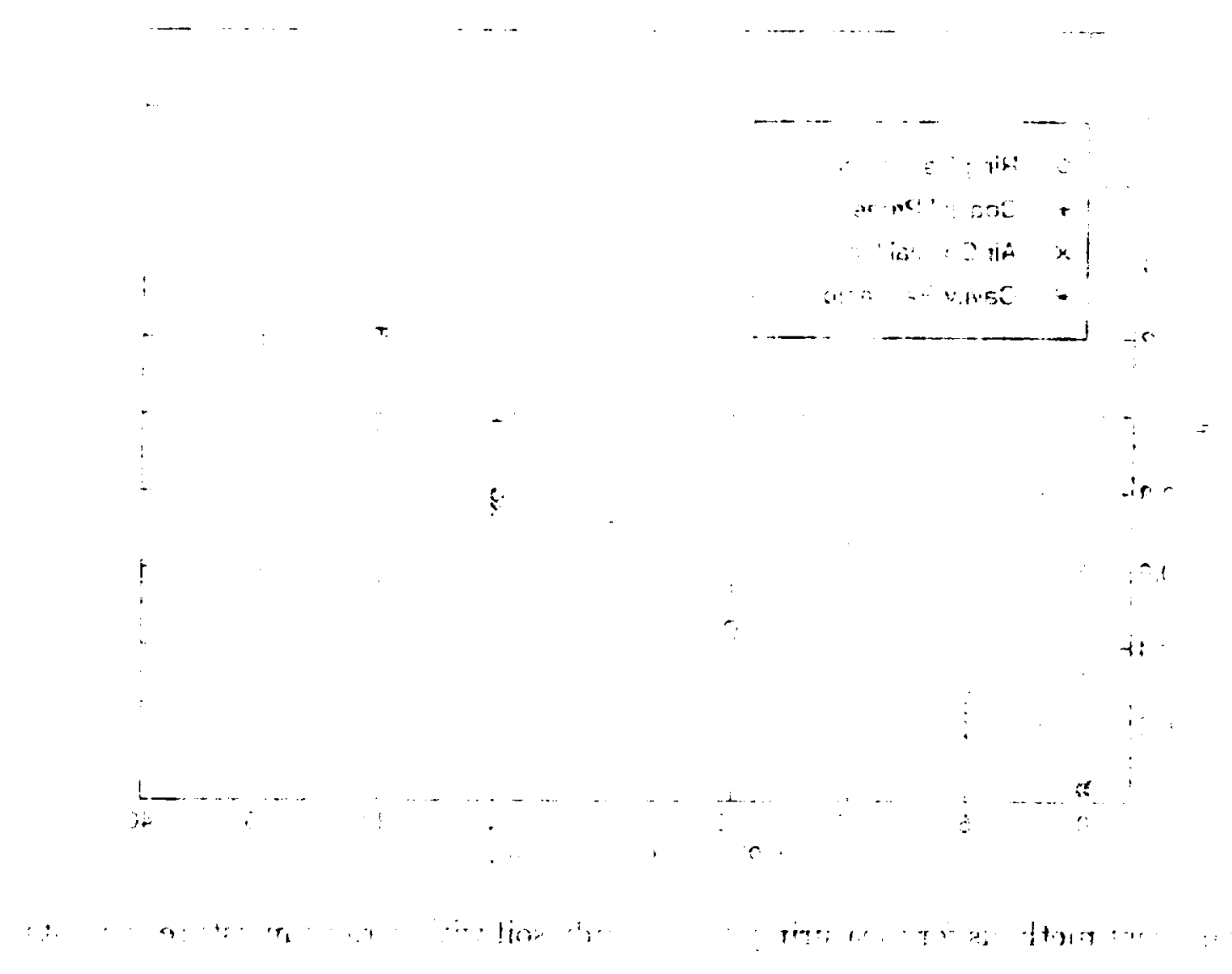

